\title{
Control del flujo de acero líquido en una artesa de colada continua de
}

\section{salidas múltiples $(\cdot)$}

\author{
Rodolfo Morales-Dávila*, José de Jesús Barreto-Sandoval**, \\ Simón López-Ramírez*** y Jorge Palafox-Ramos****
}

Resumen

Utilizando las técnicas de modelación física y matemática, se estudia en detalle el comportamiento del flujo de acero líquido en una artesa de salidas múltiples para colar tochos (bloomś). Se estudiaron tres casos: una artesa sin accesorios (SA), una artesa con dos pares de bafles y una placa de impacto ondulada (BPIO), y una artesa equipada con un inhibidor de turbulencia y un par de represas ("dams") (IT\&D). También, se simuló matemáticamente la difusión turbulenta de la mezcla química del trazador y se comparó con los resultados experimentales. La configuración IT\&D mostró una buena distribución del trazador entre las salidas y una mejora en las características del patrón de flujo reduciendo el retroflujo, logrando una buena relación de volumen mezclado y de flujo tapón. El modelo de transferencia de masa, acoplado con el modelo de turbulencia $k-\varepsilon^{\prime \prime}$, predice aceptablemente bien la mezcla química experimental del trazador en el modelo físico.

$$
\text { s.p.tin }
$$

Palabras clave Metalurgia de la artesa. Modelos físicos y matemáticos. Control del flujo de acero. Dispersión del trazador. Inhibidores de turbulencia.

\section{Flow control of molten steel in a multi-strand continuous casting tundish}

\begin{abstract}
Physical and mathematical modelling techniques were used to study the molten steel flow behaviour in a multi-strand bloom tundish. Three different cases were studied: a bare tundish (BT), a tundish equipped with two pairs of baffles and a waved impact pad (BWIP) and a tundish equipped with a turbulence inhibitor and a pair of dams (TI\&D). Chemical mixing of tracer turbulence diffusion was also simulated and compared with experimental results. The TI\&D arrangement showed a better tracer distribution among the outlets and an improvement of the fluid flow characteristics yielding lower values of back mixing flow and a good relation of mixed and plug volume flows. The mass transfer model coupled with the $k-\varepsilon$ turbulence model predicted acceptably well the experimental chemical mixing of the tracer in the water model.
\end{abstract}

Keywords Tundish metallurgy. Physical and mathematical modelling. Molten steel flow. Tracer dispersion. Turbulence inhibitors.

\section{INTRODUCCIÓN}

El control de flujo de acero en la artesa con una o dos salidas utilizando modificadores de flujo ya ha sido estudiado ampliamente usando modelos análogos de agua ${ }^{[1-8]}$. También se han reportado estudios usando modelos de agua y técnicas de simulación matemáticas para simular el flujo de acero en arte- sas de salidas múltiples para colar palanquilla y tochos (billets y blooms). Recientemente, estas técnicas se han empleado para desarrollar tecnologías innovadoras de dispositivos de control de flujo ${ }^{[9-14]}$. Uno de los desarrollos más notables son los inhibidores de turbulencia (IT), los cuales son esenciales para disminuir la turbulencia del fluido en la región de entrada a la artesa ${ }^{[15-17]}$. La bibliografía

$(\bullet)$ Trabajo recibido el día 2 de marzo de 2000 y aceptado en su forma final el 27 de julio de 2000.

(*) Profesor, Instituto Politécnico Nacional - ESIQIE, Dpto. de Ingeniería Metalúrgica y Materiales, Apdo. postal 75-874, México D.F., CP 07300 (México).E-mail: rodolfo@ragnatela.net.mx.

(**) Profesor, Instituto Tecnológico de Morelia, Centro de Graduados en Materiales, Av. Tecnológico No 1.500, Morelia, Michoacán (México). E-mail: barretos@prodigy.net.mx.

(***) Anteriormente Estudiante de Doctorado en el Instituto Politécnico Nacional - ESIQIE, Dpto. de Ingeniería Metalúrgica y Materiales. Apdo. postal 75-874, México D.F., C.P. 073000 (México). Actualmente en FOSECO Ltd. Cleveland (EE.UU.).E-mail: slopezr@infonie.fr.

$\left(^{* * *}\right)$ Estudiante de Doctorado en el Instituto Politécnico Nacional - ESIQIE, Dpto. de Ingeniería Metalúrgica y Materiales. Apdo. postal 75-874, México D.F., C.P. 07300 (México). 
ha reportado el uso de estos dispositivos de control de flujo en artesas de una o dos salidas para colar planchones (slabs) $)^{[18-20]}$.

Los inhibidores de turbulencia han mostrado ser muy útiles para evitar el atrapamiento de escoria por el metal y la absorción de oxígeno y nitrógeno del aire circundante durante el cambio de cuchara, así como para disminuir la cantidad de acero degradado durante los cambios de grado. La tendencia actual, similar a la de los otros dispositivos de control de flujo, es que los inhibidores de la turbulencia deben diseñarse y fabricarse de manera individual de acuerdo a las condiciones operativas de cada planta. Es decir, cada inhibidor de turbulencia debe diseñarse según la forma y diseño de la artesa, altura del acero, razón de flujo de acero, etc.

En el presente trabajo se estudia detalladamente el control de flujo de acero líquido usando un inhibidor de turbulencia en una artesa de cuatro salidas para colar tochos (blooms). Una artesa de salidas múltiples presenta varios desafíos, tales como poder mantener la misma temperatura de colada, una composición química homogénea y limpieza de acero similar en cada salida. Asimismo, se persiguen varios objetivos: Estudiar los efectos de un IT sobre los patrones de flujo, la difusión y la distribución del trazador en las diferentes salidas de la artesa, comparando el desempeño entre los tres casos estudiados.

Para lograr estos objetivos se utilizaron las técnicas de modelación física y matemática permitiendo obtener conclusiones útiles en forma complementaria y simultánea.

\section{MODELACIÓN FÍSICA}

Usando el criterio de similitud de Froude, se diseñó y construyó un modelo físico a escala de $1 / 3$, en acrílico transparente con un espesor de 0,0127 m. La figura 1 muestra las dimensiones geométricas del modelo. Como puede verse, las posiciones de las salidas son asimétricas con respecto al eje central de la artesa.

Se estudiaron tres diferentes configuraciones, la primera es simplemente una artesa sin accesorios, la segunda una artesa con modificadores de flujo que consiste en dos pares de bafles y una placa de impacto ondulada (BPIO) y una tercera que consiste en un IT y un par de represas (dams) (IT $\dot{\&} D)$.

El diseño de los bafles altos y bafles bajos para la configuración de BPIO se muestra en la figura $2 \mathrm{a} \mathrm{y} \mathrm{b}$, respectivamente. En la figura $2 \mathrm{c}$ se muestra el diseño de la placa de impacto ondulada. De
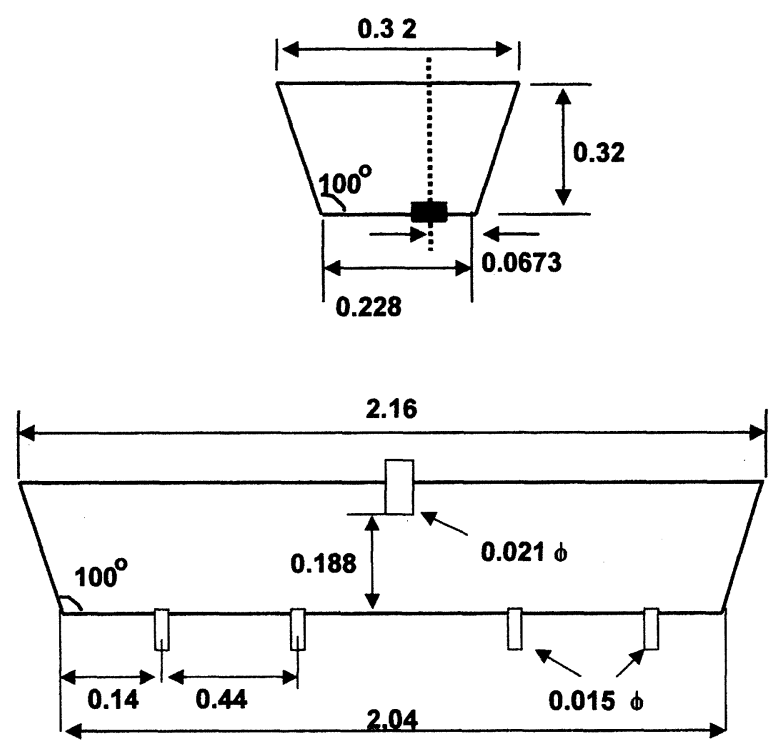

Figura 1. Dimensiones geométricas del modelo de agua (m).

Figure 1. Geometric dimensions of the water model $(\mathrm{m})$.

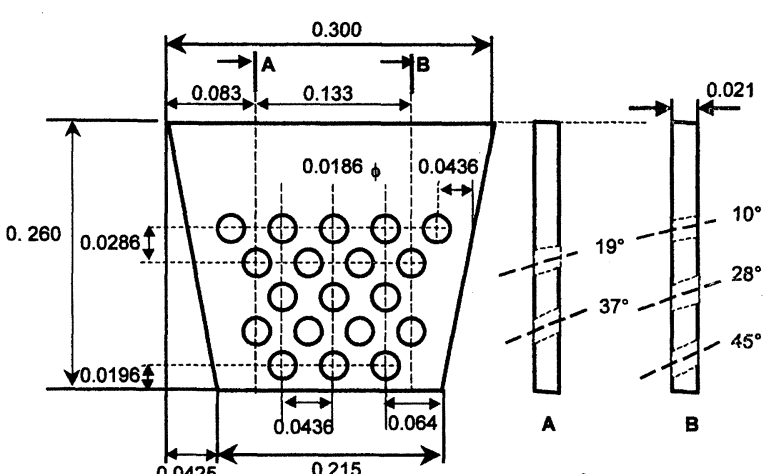

a)
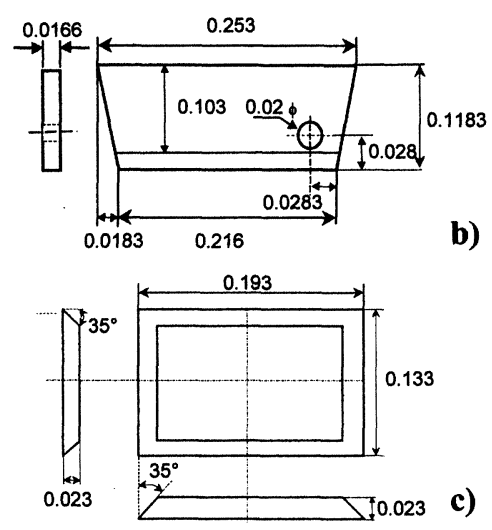

Figura 2. Dimensiones geométricas de la configuración BPIO (m). a) Bafle alto. b) Bafle bajo. c) Placa de impacto ondulada.

Figure 2. Geometric dimensions for the BWIP tundish arrangement (m). a) Tall baffles. b) Short baffles. c) Waved impact pad. 
forma semejante, la configuración de IT\&D se muestra en la figura $3 a$ y b. La primera muestra el diseño de las represas y la segunda del IT.

En la figura 4 a se muestran las posiciones de los dos pares de represas dentro de la artesa para la configuración de BPIO, y en la figura $4 \mathrm{~b}$ la de $I T \& D$, en las vistas verticales respectivas. Las barras tapón mostradas realizan el control de la razón flujo de acero, utilizando una barra para cada salida. Las condiciones de operación de la artesa se reportan en la tabla I.

Las curvas de distribución de tiempos de residencia (DTR), se determinaron experimentalmente utilizando como trazador un colorante rojo que se inyectó en forma de pulso en el chorro de entrada a un tiempo igual a cero. La concentración del trazador se mide en dos salidas, a una se le llamó interior (la más cercana a la entrada del chorro) y a la otra exterior (la más cercana a la pared transversal de la artesa), usando dos espectrofotómetros de UV LAMBA 2.

La señal de salida de estos aparatos se alimenta a un ordenador personal equipado con una tarjeta de adquisición de datos, proporcionada con el software para procesar los datos reales de las mediciones; posteriormente se adimensionalizaron según procedimiento propuesto por Sahai et al. ${ }^{[21]}$

Así, suponiendo que el flujo volumétrico a través de las cuatro salidas fuera idéntico, tenemos que la cantidad de trazador inyectado en un periodo $d t$ que fluye a través de la salida "i" sería:[22]

$$
d m_{\mathrm{i}}=\mathrm{C}_{\mathrm{i}}(t) \mathrm{Q} d t
$$

donde $C_{i}$ es la concentración del trazador en la salida del chorro " $i$ ", $Q$ es el flujo volumétrico y $m_{\mathrm{i}}$ la masa del trazador a la salida "i" (ver la lista de símbolos). Si M es la masa total del trazador inyectada por impulso, entonces obtenemos la siguiente expresión:

$$
\frac{d m_{\mathrm{i}}}{\mathrm{M}}=E_{\mathrm{i}}(t) d t
$$

Tabla I. Parámetros básicos del modelo de agua

Table I. Main parameters of the water model

\begin{tabular}{ll}
\hline \multicolumn{1}{c}{ Parámetros } & \multicolumn{1}{c}{ Modelo } \\
\hline Volumen de agua para 27 $\mathrm{t}$ & $0,1475 \mathrm{~m}^{3}$ \\
Altura del agua 27 $\mathrm{t}$ & $0,2530 \mathrm{~m}$ \\
Penetración de la boquilla & $0,065 \mathrm{~m}$ \\
Caudal volumétrico & $0,020 \mathrm{~m}^{3} \mathrm{~min}^{-1}$ \\
\hline
\end{tabular}
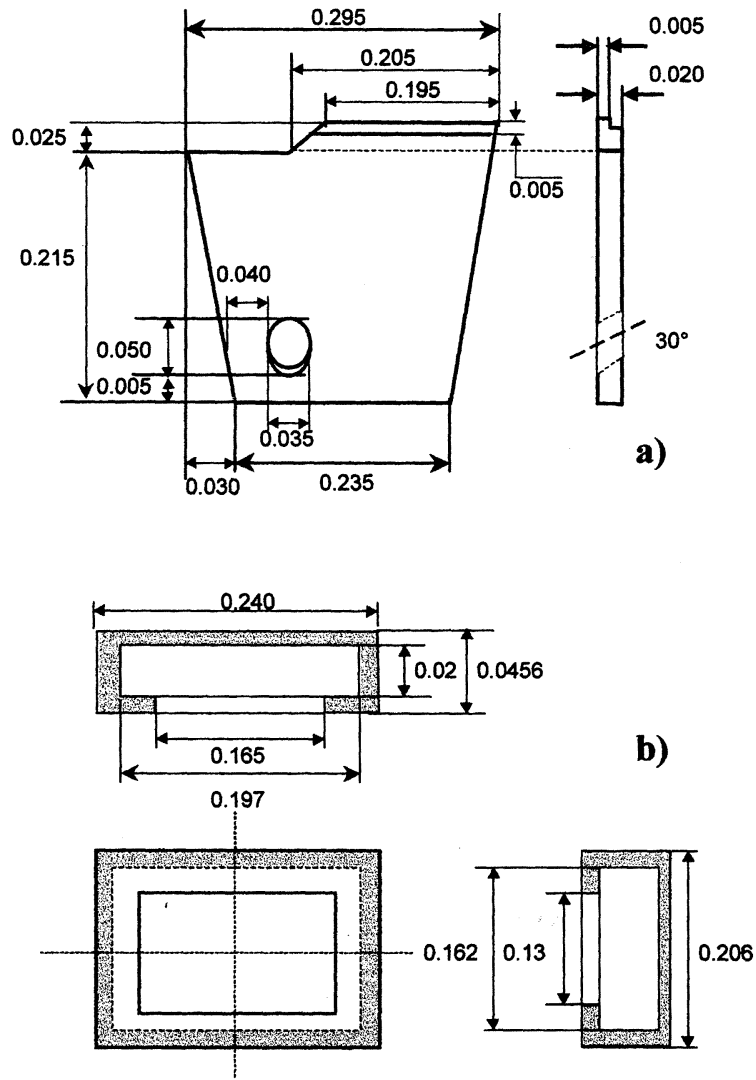

Figura 3. Dimensiones geométricas de la configuración IT\&D. a) Inhibidor de turbulencia. b) Represas (dams).

Figure 3. Geometric dimensions for the TI\&D tundish arrangement (m). a) Baffles. b) Turbulence inhibitor.

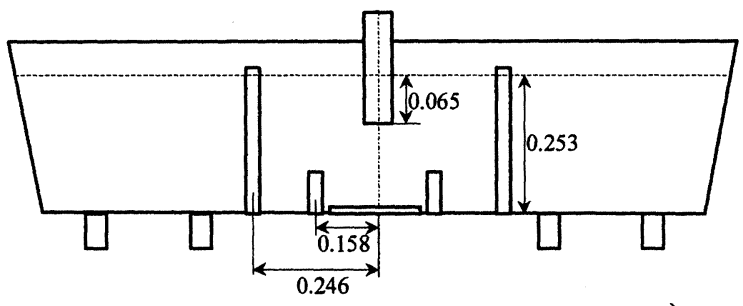

a)

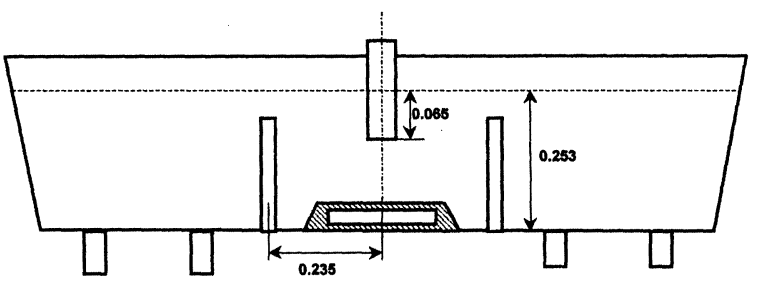

b)

Figura 4. Esquemas de las configuraciones del dsitribuidor empleado en este estudio. a) Configuración BPIO. b) Configuración IT\&D.

Figure 4. Schematics of tundish arrangements employed in this study. a) BWIP arrangement. b) TI\&D arrangement. 
Integrando esta ecuación para todas las salidas:

$$
\int_{0}^{\infty} E_{1}(t) d t+\int_{0}^{\infty} E_{2}(t) d t+\ldots . .=1,0
$$

o simplemente:

$$
\int_{0}^{\infty} E(t) d t=1,0
$$

Una vez que se obtienen las curvas de DTR experimentalmente, los parámetros de flujo pueden ser calculados utilizando los métodos discutidos en la referencia 21.

\section{MODELO MATEMÁTICO}

\subsection{Ecuaciones fundamentales}

El modelo matemático fue diseñado para simular el flujo del fluido de agua dentro del modelo análogo de la artesa y el proceso de mezclado químico del trazador inyectado por un impulso en el chorro entrante. Se consideraron dos casos, la artesa sin accesorios y la artesa con una configuración de IT\&D, por ser los más representativos del comportamiento de los patrones de flujo.

El modelo involucra la solución tridimensional (3D) simultánea de las ecuaciones de turbulencia de Navier-Stokes, la ecuación de transferencia de masa, la ecuación de continuidad y las dos ecuaciones para el modelo $k-\varepsilon$ seleccionadas para representar la viscosidad turbulenta. Las ecuaciones se redujeron a su equivalente de diferencias finitas integrando las celdas computacionales, el dominio 3D se dividió como lo muestra la figura 5. Las ecuaciones de momentum turbulento se resolvieron bajo condiciones de estado estable y la ecuación de transferencia de masa turbulenta se resolvió bajo condiciones de estado inestable. Este es un procedimiento similar al empleado en los modelos físicos, es decir, el fluido se estabiliza a una

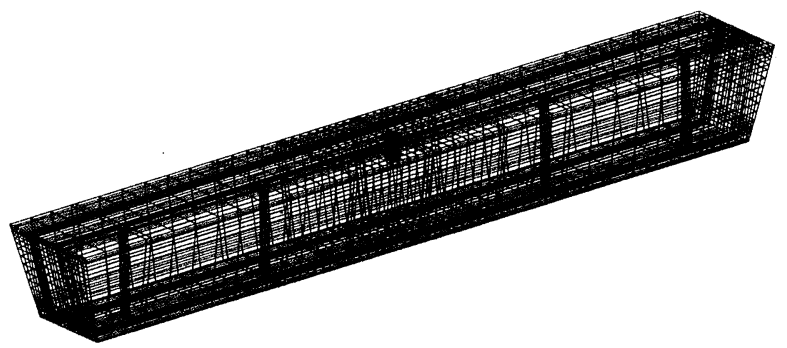

Figura 5. Malla computacional 3D empleada en el modelo matemático.

Figure 5. 3D computational mesh employed in the mathematical model. razón de flujo volumétrico constante y en un instante arbitrario tomado como cero, se inyecta el trazador iniciando el mezclado químico inestable en el fluido. Las ecuaciones fundamentales son representadas, en notación tensorial por:

La ecuación de continuidad

$$
\operatorname{div} \vec{V}=0
$$

La ecuación de momentum

$$
\begin{gathered}
\frac{\partial}{\partial t}\left(\rho u_{\mathrm{i}}\right)+\frac{\partial}{\partial x_{\mathrm{j}}}\left(\rho u_{\mathrm{i}} u_{\mathrm{j}}\right)=-\frac{\partial P}{\partial x_{\mathrm{i}}}+ \\
+\frac{\partial}{\partial x_{\mathrm{j}}}\left[\mu_{\mathrm{eff}}\left(\frac{\partial u_{\mathrm{i}}}{\partial x_{\mathrm{j}}}+\frac{\partial u_{\mathrm{j}}}{\partial x_{\mathrm{i}}}\right)\right]+\rho g_{\mathrm{i}}
\end{gathered}
$$

La ecuación de transferencia de masa

$$
\frac{\partial \mathrm{C}}{\partial t}+u_{\mathrm{i}} \cdot \nabla \mathrm{C}=\mathrm{D}_{\mathrm{eff}} \nabla^{2} \mathrm{C}
$$

La ecuación que describe la energía cinética turbulenta

$$
\frac{\partial}{\partial t}(\rho \varepsilon)+\frac{\partial}{\partial x_{\mathrm{i}}}\left(\rho u_{\mathrm{i}} k-\frac{\mu_{\mathrm{eff}}}{\sigma_{\mathrm{k}}} \frac{\partial k}{\partial x_{\mathrm{i}}}\right)=\mathrm{G}-\rho \varepsilon
$$

La ecuación que describe la proporción de dispersión de energía de la turbulencia

$$
\frac{\partial}{\partial t}(\rho \varepsilon)+\frac{\partial}{\partial x_{i}}\left(\rho u_{\mathrm{i}} \varepsilon-\frac{\mu_{\text {eff }}}{\sigma_{\varepsilon}} \frac{\partial k}{\partial x_{i}}\right)=\frac{1}{k}\left(C_{1} G-C_{2} \rho \varepsilon^{2}\right)
$$

donde

$$
G=\mu_{t} \frac{\partial u_{i}}{\partial x_{i}}\left(\frac{\partial u_{i}}{\partial x_{j}}+\frac{\partial u_{j}}{\partial x_{j}}\right)
$$

La viscosidad efectiva es la suma de la viscosidad laminar y la viscosidad turbulenta:

$$
\mu_{\text {eff }}=\mu_{1}+\mu_{\mathrm{t}}
$$

Se relaciona a la viscosidad turbulenta con la energía turbulenta por:

$$
\mu_{\mathrm{t}}=\mathrm{C}_{\mathrm{D}} \rho k^{2} / \varepsilon
$$

Los valores para las constantes $\mathrm{C}_{1}, \mathrm{C}_{2}, \mathrm{C}_{\mathrm{D}}, \sigma_{\mathrm{k}} \mathrm{y}$ $\sigma_{\varepsilon}$ en el modelo $k-\varepsilon$ son 1,$43 ; 1,92 ; 0,09 ; 1,00$ y 1,30 , respectivamente ${ }^{[23]}$.

En las ecuaciones de transferencia de masa, $D_{\text {eff }}$ es la difusividad de transferencia de masa eficiente, que es la suma de las difusividades molecular y turbulenta. La difusividad turbulenta $D_{\mathrm{t}}$ se relaciona con la viscosidad turbulenta $\mu_{\mathrm{t}}$ por 


$$
\sigma_{\mathrm{t}}=\mu_{\mathrm{t}} /\left(\rho D_{\mathrm{t}}\right)
$$

Subsecuentemente, el flujo turbulento generalmente lleva la masa de un Prandtl equivalente de mezcla, por lo que se supone que $\rho$ es igual a uno ${ }^{[24]}$. Entonces de la ecuación (13) obtenemos:

$$
D_{t}=\mu_{t} / \rho
$$

\subsection{Condiciones frontera}

Las condiciones frontera se aplican en todas las superficies sólidas, incluyendo los modificadores de flujo y paredes interiores de la artesa. Cerca de cualquier superficie sólida existe una subcapa laminar muy delgada. Entre ésta y el centro turbulento existe también una subcapa estándar que está en un estado entre el laminar y el flujo turbulento. Consecuentemente, para los nodos cerca de una pared sólida, son necesarios las funciones de pared para poder calcular los valores de una variable, ya que en esos lugares existen gradientes muy pronunciados. Si un nodo en el dominio 3D se encuentra en la subcapa laminar se asume una relación lineal entre el esfuerzo cortante sobre la pared y el gradiente de velocidad

$$
\tau_{\mathrm{w}}=\mu \frac{\Delta v}{\Delta n}
$$

Si el nodo está más allá de la subcapa laminar, se aplica la ley logarítmica para calcular el esfuerzo sobre la pared:

$$
\frac{v_{p}}{v^{*}}=\frac{1}{K_{v}} \ln \left(E y^{+}\right)
$$

donde

$$
v^{*}=\sqrt{\frac{\tau_{w}}{\rho}}
$$

y

$$
y^{+}=\rho v^{*} \Delta n_{p} / \mu
$$

donde $K_{v}$ es la constante de Von Karman $(0,42), E$ es una constante empírica $(9,81)$ y $v_{\mathrm{p}}$ es la velocidad del fluido cerca de la pared.

La condición límite para $k$ y $\varepsilon$ en esta subcapa ha sido calculada con el conocimiento previo de $y^{+}$ utilizando la expresión

$$
y^{+}=\frac{\rho k_{p}^{1 / 2} C_{\mu}^{1 / 4} \Delta n_{p}}{\mu}
$$

donde $k_{\mathrm{p}}$ es la energía cinética turbulenta en el punto de la malla cercana a la pared $\rho$ e $\Delta n_{p}$ es la distancia del punto $\rho$ a la pared. La ecuación (19) es un resultado empírico de datos de flujo turbulentos para $y^{+}$con los valores de entre 10 y 20 . Aquí la ecuación (15) se usa cuando $y^{+}$es más pequeño que 12 y la ecuación (19) se usa cuando $y^{+}$ es más grande que este valor.

En la superficie libre del baño y en los planos de simetría los gradientes de velocidad y flujos de masa, así como los gradientes de la energía cinética turbulenta y los gradientes de disipación de energía cinética se iniciaron igual a cero.

En el chorro de entrada, el perfil de flujo se supone plano y fue calculado por

$$
U_{\text {in }}=Q / A_{\text {nozzle }}
$$

Los valores iniciales para $k$ y $\varepsilon$ a la entrada se calcularon con las ecuaciones siguientes:

$$
\begin{gathered}
k_{\text {in }}=0,01 U_{\text {in }}^{2} \\
\varepsilon_{\text {in }}=2 k_{\text {in }}^{3 / 2} / D_{\text {nozzle }}
\end{gathered}
$$

\subsection{Condiciones iniciales}

Las ecuaciones (5), (6), (8) y (9) se resolvieron junto con sus condiciones límite que usan las expresiones auxiliares, explicadas anteriormente, hasta alcanzar el estado estacionario del flujo. El perfil de velocidad calculado se empleó posteriormente para resolver la ecuación (7) para la concentración del trazador bajo las condiciones de estado inestable. Aquí, se supone implícitamente que la presencia del trazador no afecta a la densidad del agua en una magnitud apreciable. La condición inicial para resolver la ecuación (7) se declara como sigue:

$$
t=0 \quad y x_{0}, y_{0}, z_{0} C=\frac{M}{V_{\text {nozzle }}}
$$

donde $M$ es la masa total del trazador y $V$ es el volumen de la columna de agua en la buza de la cuchara desde el punto de inyección a la punta de la buza, la cual se supone que encuentra un mezclado perfecto y $\mathrm{x}_{0}, \mathrm{y}_{0} \mathrm{y} \mathrm{z}_{0}$ son las coordenadas de la punta de la buza en el dominio 3D.

\subsection{Solución numérica}

Las ecuaciones de continuidad, momentum y de transferencia de masa, así como las condiciones 
iniciales y todas las condiciones de frontera se reescriben en un esquema de diferencias finitas usando la malla 3D mostrada en la figura 5. Se emplea una malla densa cerca del fondo y cerca de las paredes de la artesa, sólo para evitar inconsistencias cuando se aplica la función de la pared como una condición frontera. Una malla densa también se emplea en los planos longitudinales de salida, así como en la proximidad de las barras tapón. El número total de celdas en el dominio 3D sumó 80.000 , lo que asegura cálculos exactos y confiables del flujo del fluido y transferencia de masa.

El algoritmo numérico que resuelve estas ecuaciones es conocido como PISO ${ }^{[25}$ y 26$]$ (pressure implicit with splitting operations). Este es un procedimiento de marcha de tiempo en que para cada intervalo de tiempo, una predicción es seguida por uno o más correctores usando un fraccionador no iterativo de operaciones de las ecuaciones discretizadas de continuidad, momentum, energía cinética, razón de disipación de energía cinética y de presión. De esta manera, los campos de velocidad al final de cada. intervalo son aproximaciones cercanas de las ecuaciones de cambio turbulento. Se estableció un criterio de convergencia, el cual consiste en que cuando la suma de todos los residuales para las variables es menor de $10^{-6}$ se logra la convergencia. Primeramente, se calcularon los perfiles de velocidad en estado estable y más tarde se emplearon para resolver la ecuación de transferencia de masa.

El modelo matemático se ejecutó en una estación de trabajo Silicon Graphics $\mathrm{O} 2$ con procesadores R-10000 del Laboratorio de Simulación de Procesamiento de Materiales del Departamento de Metalurgia e Ingeniería de los Materiales, ESIQIEIPN. Los resultados numéricos se almacenaron en discos compactos para, posteriormente, llevarlos a un formato especial para poder realizar los gráficos, mediante el software comercial conocido como Tecplot.

\section{RESULTADOS Y DISCUSIÓN}

\subsection{Experimentos en el modelo de agua}

La figura 6a, b y c muestra las curvas de DTR obtenidas experimentalmente para la artesa sin modificadores de flujo y la artesa con las cofiguraciones BPIO e IT\&D, respectivamente. Las curvas reportan las señales de la salida de los flujos interiores y exteriores. La artesa sin modificadores muestra una distribución desigual del trazador en ambas salidas
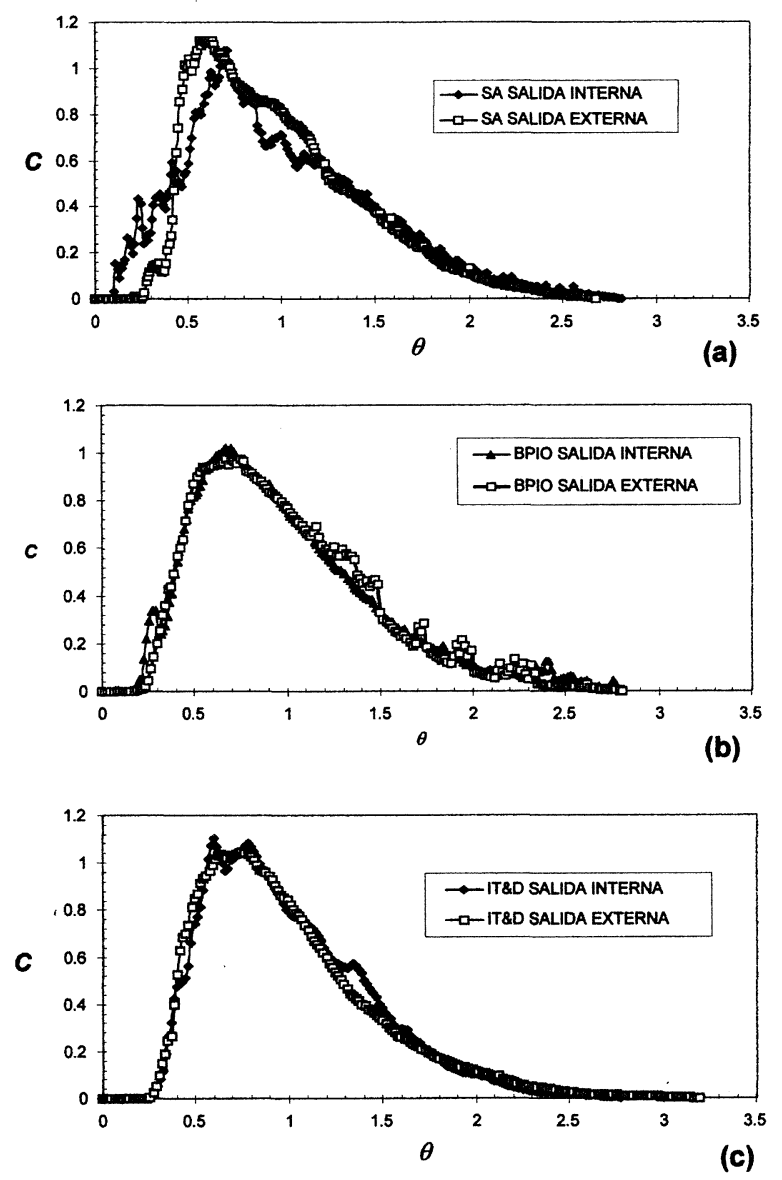

Figura 6. Curvas experimentales de DTR obtenidas en las configuraciones. a) Sin accesorios. b) Bafles y placa de impacto ondulada. c) Inhibidor de turbulencia y represas.

Figure 6. Experimental RTD curves for the tundish arrangements. a) Bare tundish. b) Baffles and waved impact pad. c) Turbulence inhibitor and dams.

y el tiempo mínimo de residencia es menor en la salida interior que en la exterior. La cresta de la concentración es más alta en la salida exterior y la diferencia entre el tiempo de la concentración máxima y el tiempo de residencia mínimo es menor en esta salida. Es evidente, como lo muestra la figura $6 a$, que en la salida interior se observa una dispersión de datos mayor para la concentración del trazador con algunas características de flujo de cortocircuito.

Usando la configuración de BPIO, las curvas de DTR muestran mejoras considerables (Fig. 6b). Aunque aún existe diferencia entre los tiempos mínimos de residencia cronometrados para ambas salidas, la diferencia es menor que en el caso precedente. El tiempo mínimo de residencia para la salida interior permanece más pequeño que el exterior. Ambas curvas muestran una dispersión estadística similar y la de la salida exterior muestra una 
forma irregular después de la cresta de la concentración. Este fenómeno se mostró de forma consistente en todos los ensayos experimentales y se observó visualmente que su origen se debe al hecho de que los agujeros en los bafles promovieron el paso del trazador en forma de paquetes. También se observó visualmente una intensa turbulencia en la superficie de baño sobre la zona de los bafles.

La figura 6 c muestra las curvas obtenidas con la configuración de IT\&D. Se puede apreciar que los patrones de flujo mejoran, que los tiempos mínimos de residencia cronometrados en ambas salidas son prácticamente iguales, así como los valores de la concentración máxima. Estos valores, también son más altos que la concentración máxima alcanzada por la configuración de BPIO. Una ventaja adicional de la configuración de IT\&D sobre la del BPIO es que emplea sólo tres piezas en el interior de la artesa en lugar de cinco. Además, la turbulencia en la superficie de baño en la zona del IT disminuye considerablemente comparada con los dos casos anteriores.

La tabla II muestra un resumen de la cuantificación de los parámetros del flujo, usando la ecuación (3) y siguiendo el procedimiento propuesto por Sahai et al. ${ }^{[21]}$ para los tres casos estudiados. Estos resultados muestran las ventajas metalúrgicas y operacionales de usar los inhibidores de la turbulencia. De acuerdo con estos resultados, su empleo en artesas de salidas múltiples ayuda a obtener composiciones químicas de acero homogéneas. Implícito con esto, hay también la posibilidad de obtener temperaturas de colada uniforme y limpieza de acero constante en cada una de las salidas de la artesa. Esto permite abrir los campos de aplicación para los inhibidores de turbulencia en máquinas de colada continua que buscan mejorar la calidad de sus productos a través de un mejor control de flujo, distribuyendo el acero líquido a todas las salidas con una temperatura, composición y limpieza uniforme.
La posición y el diseño de las represas en la configración de IT\&D, desempeñan un papel determinante para controlar la distribución del trazador en ambas salidas. Se espera que este patrón de flujo se dirija hacia la superficie libre del baño como se muestra esquemáticamente en la figura 7, donde se observa que el fluido golpea el borde superior de la represa y modifica su trayectoria, la cual sufre un impulso hacia arriba. Esto permite al trazador seguir una trayectoria más larga, aumentando su tiempo de residencia dentro de la artesa.

La figura $8 \mathrm{a}$ y $\mathrm{b}$ muestran las curvas de DTR para las salidas interiores y exteriores cuando las posiciones de las represas se encuentran a 0,23 y $0,24 \mathrm{~m}$ de la boquilla de la entrada, respectivamente. Como se puede apreciar, es diferente a las curvas de DTR cuando los represas se localizan a 0,235 m de la boquilla de la entrada (Fig. 6c); estas nuevas posiciones cambian la distribución del trazador apreciablemente en ambas salidas. Sin embargo, debe decirse que las características de flujo para las salidas en ambos casos permanecen

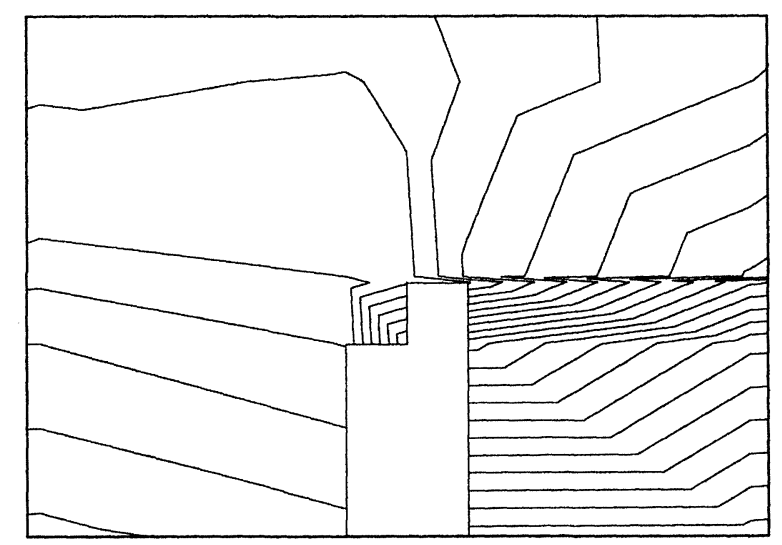

Figura 7. Representación esquemática obtenida con la simulación matemática del perfil de flujo que pasa por el borde superior de la represa.

Figure 7. Schematic representation of the flow behavior using a step in the upper side of the dam.

Tabla II. Cuantificación de las fracciones de volumen de las curvas de DTR esperimentales

Table II. Volume fractions calculated from the experimental RTD curves

\begin{tabular}{lcccccc}
\hline \multicolumn{1}{c}{ Configuraciones } & $\mathrm{V}_{\text {Muerto }}$ & $\mathrm{V}_{\text {Pistón }}$ & $\mathrm{V}_{\text {Mezclado }}$ & $\mathrm{t}_{\text {calc }}$ & $\sigma_{\theta}{ }^{2}$ & $\mathrm{D} / \mathrm{UL}$ \\
\hline $\begin{array}{l}\text { Sin accesorios (SA) } \\
\begin{array}{l}\text { Bafles + placa de impacto } \\
\text { ondulada (BPIO) }\end{array}\end{array}$ & 0,075 & 0,207 & 0,717 & 304 & 0,217 & 0,123 \\
$\begin{array}{l}\text { Inhibidor de turbulencia + represas } \\
\text { (IT\&D) }\end{array}$ & 0,105 & 0,276 & 0,618 & 362 & 0,242 & 0,141 \\
\hline
\end{tabular}



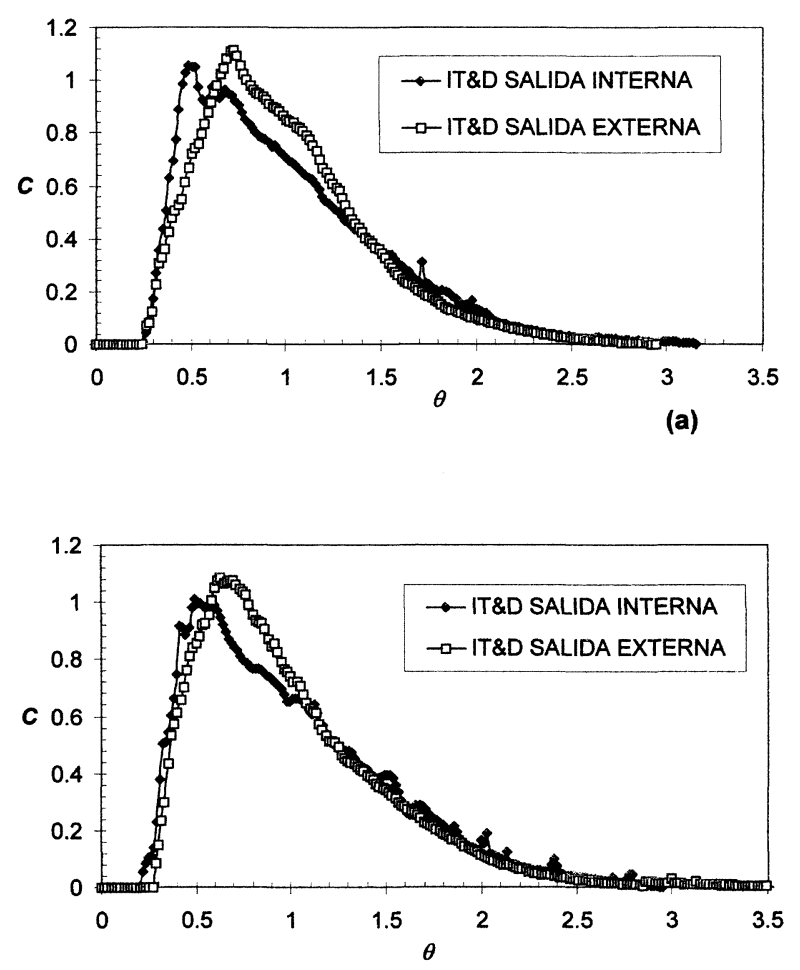

(b)

Figura 8. Curvas experimentales de DTR para dos distancias entre la represa y la boquilla de la entrada que usa la configuración IT\&D. a) 0,23 m. b) 0,24 m.

Figure 8. Experimental RTD curves for two distances between the dam and the entry nozzle using the TI\&D arrangement. a) $0.23 \mathrm{~m}$. b) $0.24 \mathrm{~m}$.

superiores a las correspondientes a la artesa sin accesorios y a la artesa con la configración de BPIO (compárense las Figs. 6a y b con las Figs. 8a y b).

\subsection{Modelo matemático del flujo del fluido}

La figura 9a y b muestra el perfil de velocidad de acero en el plano de entrada y en un plano localizado entre las salidas interna y externa en la artesa sin accesorios, respectivamente; mientras que la figura 9c y d lo hacen para los mismos planos en la artesa con la configuración de IT\&D. En el plano de entrada de la artesa sin accesorios, el fluido presenta un flujo de recirculación con velocidades más altas, dirigidas hacia la superficie superior del baño cerca de la pared después de golpear el fondo de la artesa (Fig. 9a). En el mismo plano, el IT controla la turbulencia, y el flujo de recirculación permanece, pero con velocidades menores, dirigido hacia el fondo de la artesa localizado cerca de las paredes (Fig. 9c). El plano entre las salidas del modelo muestra una recirculación del flujo como una influencia de la alta turbulencia promovida por la alta velocidad de entrada del fluido con la

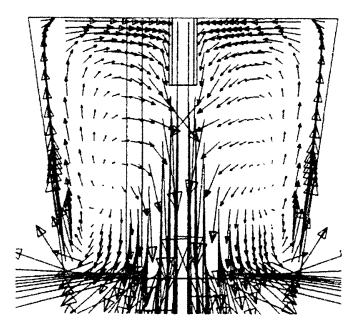

a)

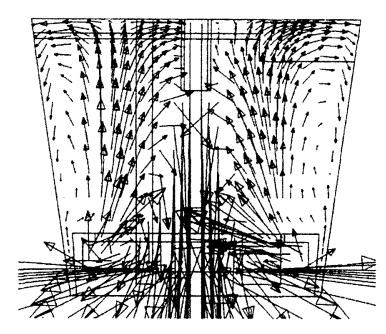

c)

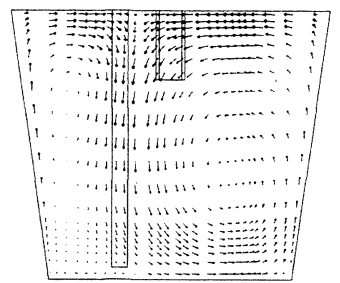

b)

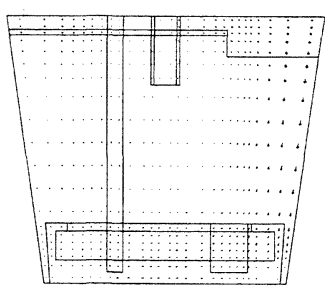

d)
Figura 9. Perfiles de velocidad del modelo de agua en el distribuidor. a) Plano a la entrada en la configuración SA. b) Pláno localizado entre ambas salidas en la configuración SA. c) Plano a la entrada en la configuración IT\&D. d) Plano localizado entre ambas salidas en la configuración IT\&D.

Figure 9. Velocity fields of water in the tundish model. a). Entry plane in the BT arrangement. b) Plane located between both outlets in the BT arrangement. c) Entry plane in the TI\&D arrangement. d) Plane located between both outlets in TI\&D arrangements

artesa sin accesorios (Fig. 9b). El patrón de flujo correspondiente usando la configuración de IT\&D mostrado en la figura $9 \mathrm{~d}$ indica que la recirculación del flujo se ha eliminado, indicando que el mezclado transversal disminuye.

Las características de flujo de agua en los planos de ambas salidas (interior y exterior) se muestran en las figuras $10 \mathrm{a}-\mathrm{d}$. La figura $10 \mathrm{a}$ muestra un flujo asimétrico fuertemente recirculado debido a la posición de las salidas, indicado por la barra tapón, en el plano de la salida interior de la artesa sin accesorios. Esencialmente, los mismos rasgos arriba expresados para el plano de la entrada también se observan aquí. Las velocidades del fluido mayores se dirigen hacia la superficie superior libre del baño cerca de las paredes de la artesa. En la salida externa se forma el mismo patrón de flujo, aunque los vectores de velocidad son más pequeños.

Para una configuración IT\&D, los vectores de velocidad no presentan recirculación, como puede verse en las características de flujo para las salidas interiores y exteriores mostradas en las figuras $10 \mathrm{c}$ $\mathrm{y} d$ respectivamente. Los patrones de flujo son muy similares en ambas salidas. 


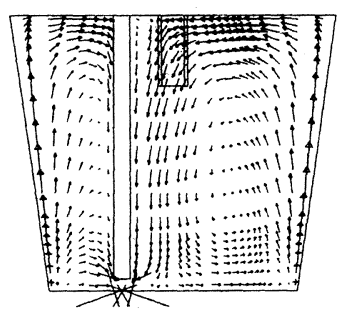

a)

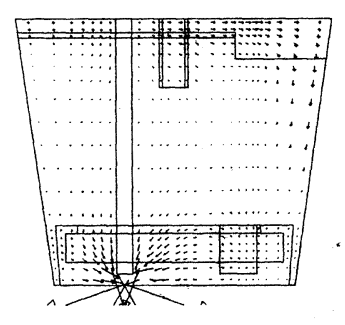

c)

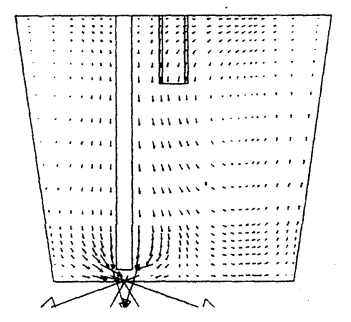

b)

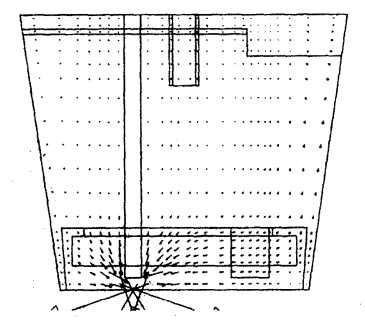

d)
Figora 10. Perfiles de velocidad del modelo de agua en el distribuidor. a) Salida interna en la configuración SA. b) Salida externa en la configuración SA. c) Salida interna en la configuración IT\&D. d) Salida externa en la configuración IT\&D.

Figure 10. Velocity fields of water in the tundish model. a) Internal outlet in BT arrangement. b) External outlet in BT arrangement. c) Internal outlet in TI\&D arrangement. d) External outlet in TI\&D arrangement.

La dispersión más alta observada en la figura $6 \mathrm{a}$ para la artesa sin accesorios, comparada con la configuración de IT\&D (Fig. 6c) y cuantificada en la tabla II, puede explicarse con las figuras 9 y 10 como sigue:

En la artesa sin accesorios, el patrón de flujo de recirculación se mantiene desde el plano de entrada hasta, por lo menos, la salida externa promoviendo un mezclado transversal, además del mezclado longitudinal (Figs. 11a y c, y 12a y c). Entretanto, usando una artesa con la configuración de IT\&D, el mezclado transversal sólo se observa en la zona de entrada, y después la represa hace que el fluido se comporte como un flujò tapón.

La figura 11a-d muestra los perfiles de velocidad presentes en los planos horizontales; la figura 11a muestra el perfil de velocidad de acero en la superficie del baño en una artesa sin accesorios, y la figura $11 \mathrm{~b}$ muestra el perfil correspondiente en la artesa con una configuración de IT\&D. La figura 11c y d muestra el mismo tipo de información para un plano localizado a la mitad de la altura del baño.

Los perfiles de velocidad verticales-longitudinales se muestran en la figura 12a-d. La figura 12a muestra el perfil del plano de salida para la artesa sin accesorios, y la figura $12 \mathrm{~b}$ muestra el perfil correspondiente

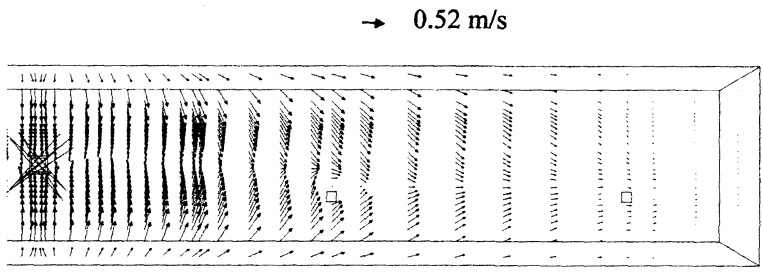

a)

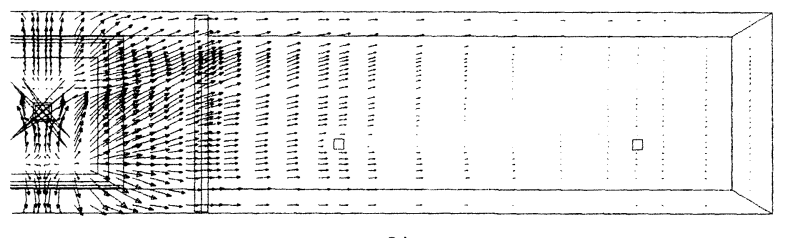

b)

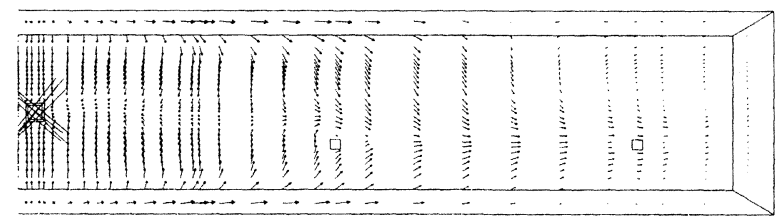

c)

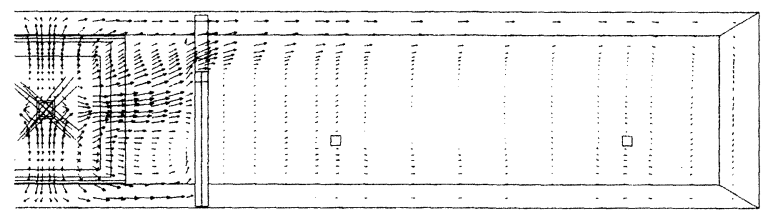

d)

Figura 11. Vistas superiores de los campos de velocidad en el modelo de agua. a) Vista superior del baño para SA. b) Vista superior del baño para IT\&D. c) Plano del centro del baño para SA. d) Plano del centro del baño para IT\&D.

Figure 11. Upper views of the velocity fields of water in the tundish model. a) Upper bath surface in BT arrangement. b) Upper bath surface in TI\&D arrangement. c) Center plane of the bath height in BT arrangement. d) Center plane of the bath height in TI\&D arrangement.

para la artesa con la configuración de IT\&D. La figura 12c y d muestra el mismo tipo de información para planos localizados cerca de la pared.

Las figuras 10,11 y 12 indican la naturaleza tridimensional del flujo. El flujo también es asimétrico en los planos horizontales debido a la posición asimétrica de las salidas. En la artesa sin accesorios, la entrada del fluido golpea el área de la placa de impacto provocando salpicaduras (Fig. 12a), mientras en la artesa con la configuración de IT\&D existe la formación de un flujo de recirculación, pero los vectores de velocidad son pequeños debido a la influencia del inhibidor (Fig. 12b). Cerca de la pared, las velocidades del fluido son altas y se dirigen hacia arriba en un plano localizado cerca de la pared (Fig. 12c) en la artesa sin accesorios. Para una artesa con una configuración de IT\&D, las velocidades son considerablemente bajas y se dirigen ligeramente hacia el fondo de la artesa (Fig. 12d). 
$\rightarrow 0.52 \mathrm{~m} / \mathrm{s}$

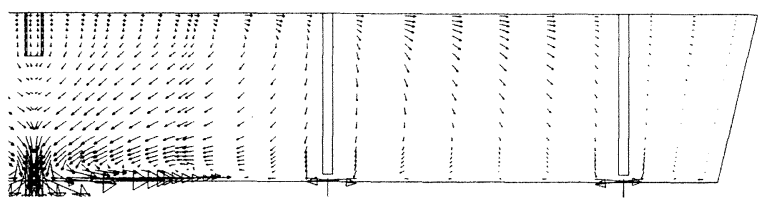

a)

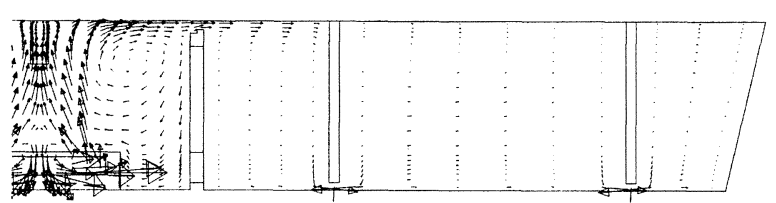

b)

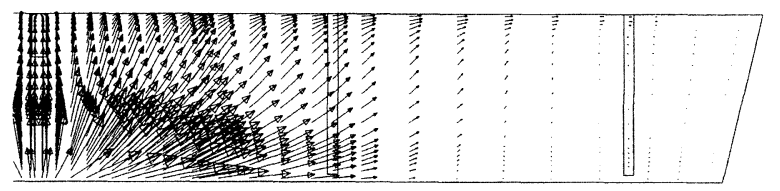

c)

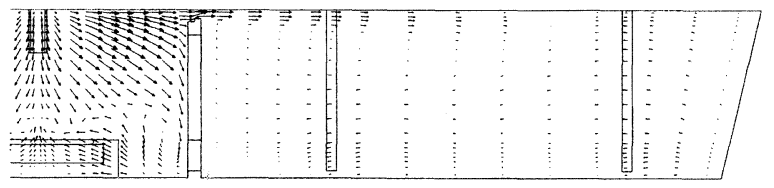

d)

Figura 12. Vistas frontales de los campos de velocidad en el modelo de agua. a) Plano a la salida en la configuración SA. b) Plano a la salida en la configuración IT\&D. c) Plano cerca de la pared en la configuración SA. d) Plano cerca de la pared en la configuración IT\&D.

Figure 12. Front view of the velocity field of water in the tundish model. a) Outlet plane in BT arrangement. b) Outlet plane in TI\&D arrangement. c) Near wall plane in BT arrangement. d) Near wall plane in TI\&D arrangement.

\subsection{Modelo matemático de transferencia de masa}

La figura 13a y b muestra la dinámica del mezclado químico del trazador, después de $30 \mathrm{~s}$ de su inyección en la boquilla de la cuchara, como líneas de isoconcentración expresadas en unidades de fracción masa para los planos longitudinales-verticales. Estas figuras corresponden al plano de entrada del fluido para una artesa sin accesorios y una artesa con la configuración de IT\&D, respectivamente. Se ve que en este tiempo corto, el trazador se ha dispersado alcanzando la salida interior completamente, y parcialmente la exterior, en la artesa sin accesorios. En la artesa con la configuración de $I T \& D$, el trazador permanece en un proceso de mezclado en la zona de entrada y el trazador ha rebasado simplemente por encima del lado superior de la represa. La figura 13 c y d muestra el mismo tipo de información para el plano de la salida en

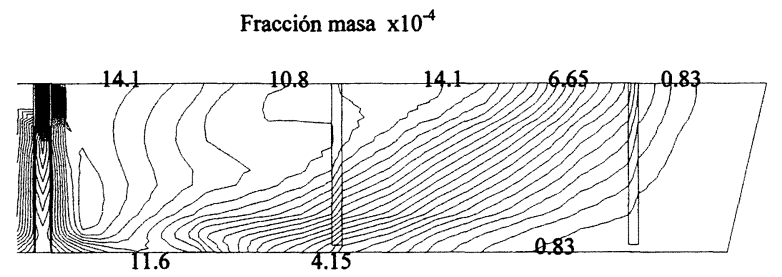

a)

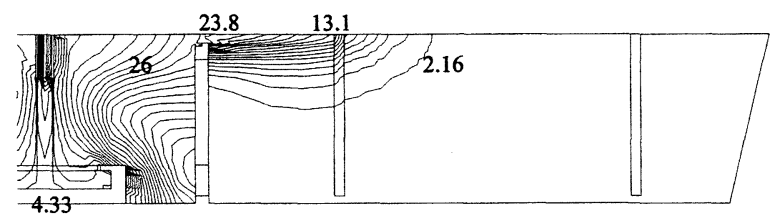

b)

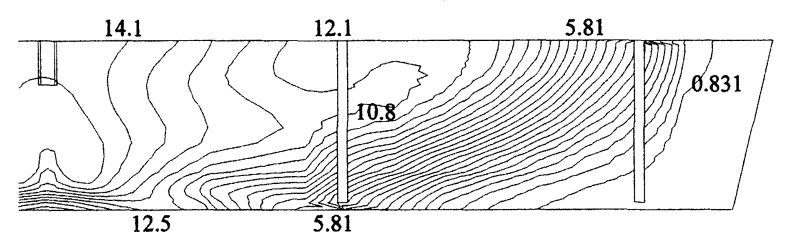

c)

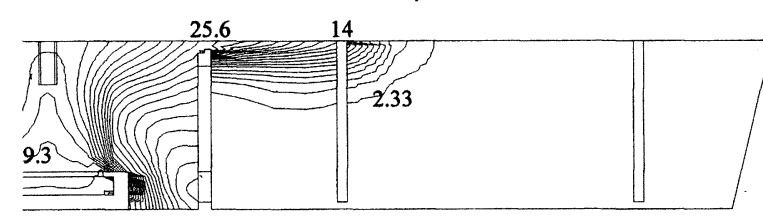

d)

Figura 13. Vista frontal de isolíneas de concentración a 30 s después de la inyección del trazador. a) Plano de la entrada en la configuración SA. b) Plano de la entrada en la configuración IT\&D. c) Plano de la salida de corriente en SA. d) Plano a la salida de corriente en IT\&D.

Figure 13. Front view of the iso-concentration lines of tracer at 30 s. After the injection. a) Inlet plane in BT arrangement. b) Inlet plane in TI\&D arrangement. c) Outlet plane in $B T$ arrangement. d) Outlet plane in TI\&D arrangement.

ambos tipos de artesas, donde los mismos comentarios son aplicables.

La figura $14 \mathrm{a}-\mathrm{d}$ muestra las vistas horizontales del mezclado químico del trazador después de $30 \mathrm{~s}$ de su inyección. La figura 14a y b muestra las líneas de isoconcentración en un plano localizado cerca del fondo de la artesa, para el caso sin accesorios y para configuración de IT\&D, respectivamente. En el primer caso, el trazador ha alcanzado ya la posición de la salida interior y ha rebasado poco más de la mitad de la trayectoria a la salida exterior. En el segundo caso, el trazador está justamente saliendo del agujero en la represa antes de llegar a la salida exterior.

La figura $14 \mathrm{c}$ y d muestra el mezclado químico del trazador, durante el mismo tiempo, en los planos superiores de una artesa sin accesorios y la artesa de IT\&D, respectivamente. En este momento (30 s después de la inyección del trazador), la dispersión ha alcanzado la pared lateral de la artesa sin accesorios, ya que la transferencia de 


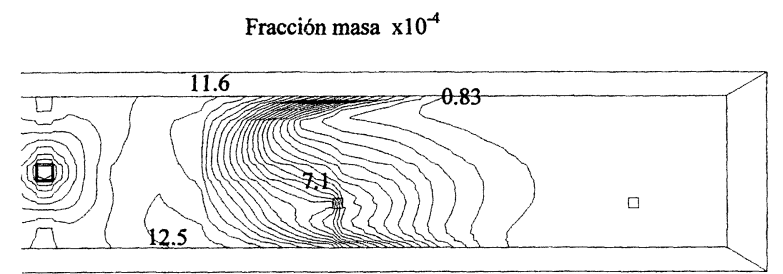

a)

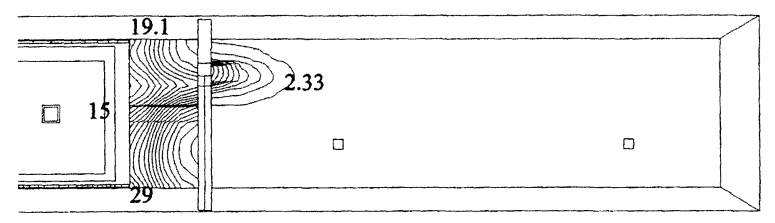

b)

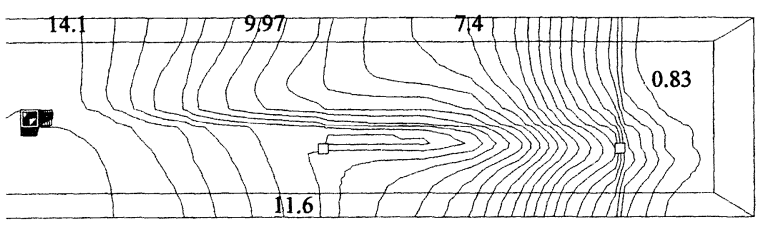

c)

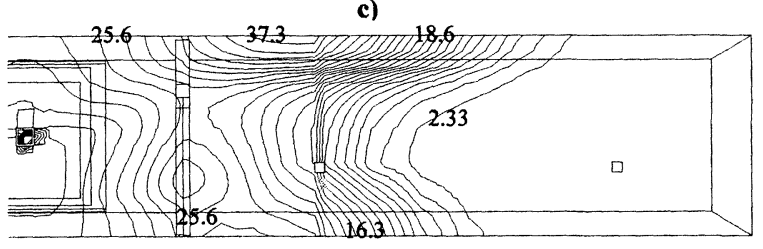

d)

Figura 14. Vista superior de isolíneas de concentración a $30 \mathrm{~s}$ después de la inyección del trazador. a) Plano cerca del suelo en la configuración SA. b) Plano cerca del suelo en la configuración IT\&D c) Plano del centro del baño en la configuración SA. d) Plano del centro del baño en la configuración IT\&D.

Figure 14. Upper view of the iso-concentration lines of the tracer at $30 \mathrm{sec}$. After the injection. a) Near the floor plane in BT arrangement b) Near the floor plane in TI\&D arrangement. c) Center plane of the bath height in BT arrangement. d) Center plane of the bath height in TI\&D arrangement.

momentum y la turbulencia son suficientemente altas para promover los procesos de mezclado transversales y longitudinales. En la configuración de IT\&D, el trazador se dirige primeramente a la superficie del baño, pero con una menor turbulencia, y las líneas de la isoconcentración están deformadas por la presencia de las salidas. El trazador todavía está lejos de la pared lateral de la artesa como puede verse en la figura $14 \mathrm{~d}$.

Los resultados de las simulaciones matemáticas para las curvas de DTR totales, esto es, la suma de todas las salidas, se muestran en la figura 15 para la artesa sin accesorios y una artesa con una configuración de IT\&D. Las curvas simuladas pueden compararse con las curvas experimentales mostradas en la figura 16; donde como se observa, existe una excelente concordancia entre las curvas calculadas y las experimentales para ambas salidas. Esto

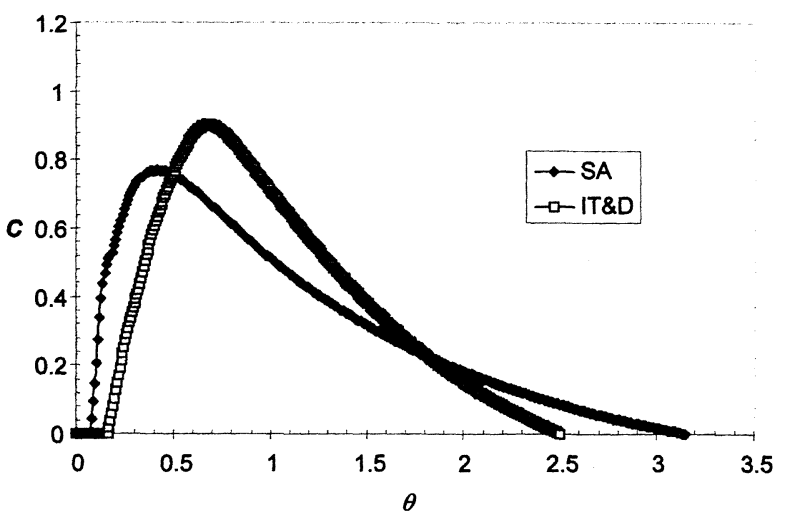

Figura 15. Curvas de DTR totales calculadas matemáticamente.

Figure 15. Mathematically calculated total RTD curves.

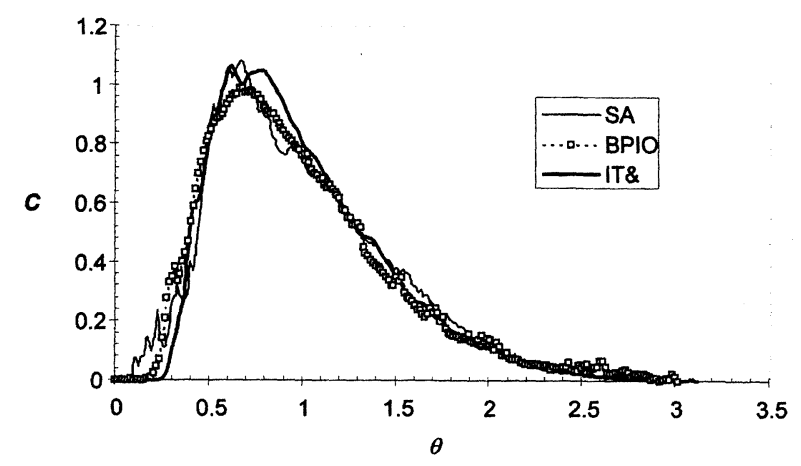

Figura 16. Curvas de DTR totales obtenidas experimentalmente.

Figure 16. Experimental total RTD curves.

demuestra que el modelo de transferencia de masa es el que describe adecuadamente el mezclado químico turbulento del trazador dentro del modelo de agua para una artesa sin accesorios y una artesa con configuraciones complejas en su interior.

Del análisis de las figuras 9 a 14 y las observaciones visuales durante los ensayos experimentales sobre la dispersión del trazador en la artesa con la configuración de IT\&D pueden hacerse las siguientes observaciones:

Un poco después de la inyección, el trazador se dirige hacia la superficie del baño sin una turbulencia apreciable. En un tiempo intermedio después de la inyección, el trazador golpea la represa y se redirige de nuevo hacia la superficie y se mantiene fluyendo a lo largo de la artesa. Cuando la dispersión del trazador llega a una distancia entre ambas salidas, el fluido ha perdido la velocidad adquirida y el trazador desciende hacia el fondo de la artesa. Una vez allí, el trazador se dispersa hacia las salidas obteniendo de esta manera una masa muy similar del trazador en ambas salidas. Además, el 
agujero de las represas que se localizan en el lado opuesto de las salidas, elimina eficientemente la zona muerta que se formaría cerca de la salida.

Estas características de flujo no se observan en la artesa sin accesorios, ni tampoco en la artesa con una configuración de BPIO, logrando como resultado final un control de flujo muy bueno con la configuración de IT\&D.

\section{CONCLUSIONES}

Se han aplicado las técnicas de modelación de agua y matemáticas para estudiar el flujo de acero líquido en una artesa de salidas múltiples para colar tocho (bloom) y las conclusiones derivadas de los resultados son las siguientes:

- El uso de la configuración consistente en un inhibidor de turbulencia y un par de represas es más eficiente para aumentar el porcentaje de volumen de flujo tapón que una artesa sin accesorios o una artesa que usa dos pares de bafles y una placa de impacto ondulada.

- Con la combinación de un inhibidor de turbulencia y un par de represas, las curvas de DTR son muy similares para las cuatro salidas de esta artesa. Además, en principio, los efectos térmicos conllevarían a una mayor limpieza del acero y temperaturas de salida homogéneas en las cuatro salidas de la artesa prototipo.

- La combinación del inhibidor de turbulencia con un par de represas disminuye considerablemente el mezclado transversal y axial del fluido, obteniendo valores bajos de retromezclado de flujo comparado con una artesa sin accesorios y una artesa con dos pares de bafles y una placa de impacto ondulada.

- Estos resultados muestran que una configuración compuesta de un inhibidor de turbulencia y un par de represas tiene un mejor desarrollo que una la configuración compleja.

- El modelo matemático usando el modelo de turbulencia $k-\varepsilon$ simuló aceptablemente bien la dispersión química del trazador bajo las condiciones turbulentas.

\section{Agradecimientos}

Los autores desean agradecer al CONACYT y al COSNET el financiamiento otorgado para la realización de este proyecto. Asimismo, al Instituto Politécnico Nacional y al Instituto Tecnológico de Morelia por su apoyo permanente a la investigación en el área de procesos metalúrgicos.

\section{Lista de símbolos}

A : Área de la sección transversal.

C : Concentración del trazador.

$\mathrm{C}_{\mathrm{i}}$ : Concentración del trazador a las salidas " $\mathrm{i}$ ”.

$\mathrm{C}_{1}, \mathrm{C}_{2}$ y $\mathrm{C}_{\mathrm{D}}$ : Constantes del modelo de turbulencia.

E : Constante empírica: ecuación (16).

$E_{\mathrm{i}}: \quad$ Curvas de DTR a las salidas "i".

$D_{\mathrm{m}}: \quad$ Difusividad molecular del trazador.

$D_{\mathrm{t}}$ : $\quad$ Difusividad turbulenta del trazador.

$D_{\text {eff }}$ : Difusividad effectiva del trazador.

G: Generación de término: ecuación (10).

$K_{v}: \quad$ Constante de Von Karman.

$k: \quad$ Energía cinética de turbulencia.

$m_{\mathrm{i}}$ : $\quad$ Masa del trazador que sale "i".

M : $\quad$ Masa total del trazador que se inyecta a través de la boquilla de entrada.

$P$ : Presión.

Q : . Flujo volumétrico.

$t: \quad$ Tiempo.

$t_{\text {calc }}: \quad$ Tiempo calculado.

$V_{\text {Muerto }}$ : Fracción de volumen muerto.

$V_{\text {Pistóin }}$ : Fracción de volumen pistón.

$V_{\text {Mezclado: }}$ Fracción de volumen mezclado.

D/UL : Parámetro de dispersión.

$v^{*}: \quad$ Define la ecuación (17), velocidad del fluido extrapolada hasta la subcapa laminar.

$u, v, w$ : Vectores de velocidad.

$y^{+}: \quad$ Define la ecuación (18), el espesor adimensional de la subcapa.

\section{Símbolos griegos}

$\varepsilon$ : Razón de disipación de la energía cinética turbulenta.

$\mu: \quad$ Viscosidad del fluido.

$\mu_{\text {eff }}$ : Viscosidad efectiva del fluido.

$\mu_{\mathrm{t}}: \quad$ Viscosidad del fluido turbulento.

$\rho:$ Densidad del fluido.

$\sigma_{t}:$ Número turbulento de Schmidt.

$\sigma_{\varepsilon}:$ Constante del modelo $k-\varepsilon_{1}$.

$\sigma^{2}:$ Varianza.

$\tau_{\mathrm{w}}$ : Esfuerzo cortante sobre la pared.

\section{REFERENCIAS}

[1] A. Mclean, L.J. Heaslip e I.D. Somerville, Continuous Casting, Vol. 1. Warrandalle, PA. EE.UU. ISS, 1983, pp. 67-84.

[2] S. Tanaka, M. Lye, M. Salcudean y R.I.L. Guthrie, 24th Annual Conference of Metallurgist, CIM, Canadá, 1985, pp. 142-161. 
[3] Y. SAHAI Y R. AHUJA, Ironmaking Steelmaking 13 (1986) 241-247.

[4] C.S. Damle e Y. Sahal, ISIJ Int. 35 (1995) 163-169.

[5] J. J. CHEN, Proc. Steelmaking Conf. 78 (1995) 593-598.

[6] Y. SAHAI Y T. EMI, ISIJ Int. 36 (1996) 1166-1173.

[7] A.K. SINHA y VASSILICOS, Ironmaking Steelmaking 25 (1998) 387-392.

[8] L.J. HeAsLIP y J. SCHADE, Ironmaking Steelmaker 26 (1999) 33-41.

[9] J. KNOEPKe y J. Mastervich, Proc. Steelmaking Conf. 69 (1986) 777-788.

[10] M. L. Lowry e Y. SAHAl, Proc. Steelmaking Conf. 72 (1989) 71-79.

[11] K.M. Godiwala, S.K. Sinha y C.S. Sivaramkrishnan, Proc. Steelmaking Conf. 77 (1994) 703-711.

[12] M. L. LOWRY e Y. SAHAl, Iron Steelmaker 19 (1992) 81-86.

[13] S. Joo, J.W. Han y R.I.L. Guthrie, Metall. Transac. B, 24B (1993) 779-788.

[14] D. Mazumdar, G. Yamanoglu y R.I.L. Guthrie, Steel Res. 68 (1997) 293-299.

[15] S. López-Ramírez, J. Palafox-Ramos, R.D. Morales, M.A. Barrón-Meza y M. VelázQuez-Toledo, Steel Res. 69 (1998) 423-428.
[16] R.D. Morales, S. López-Ramírez, J. Palafox-Ramos y D. ZACHARIAS, ISIJ Int. 5 (1999) 455-462.

[17] S. López-Ramírez, R. D. Morales y J.A. Romero-Serrano, Numerical Heat Transfer, Parte A, 37 (2000) 68-86.

[18] P. RASmussen, Proc. Steelmaking Conf. 77 (1994) 219224.

[19] D. Bolger y K. SAYLOR, Proc. Steelmaking Conf. 77 (1994) 225-233.

[20] R.D. Morales, J. Palafox-Ramos, S. López-Ramírez, M.A. Dominguez-Crespo, C. Rincón, D. Salazar y A. DAINTON, Proc. Steelmaking Conf. 81 (1998) 325-333

[21] Y. SAHAI Y T. EMI, ISIJ Int. 36 (1996) 667-673.

[22] D. MAZUMdar y R.I.L. GUTHRIE, ISIJ Int. 39 (1999) 524547.

[23] D.B. SPALDING, Int. J. Num. Math. Eng. (4) (1972) 551561.

[24] B.E. LAUnder y D.B. SPALDING, Mathematical Models of Turbulence, Academic Press, New York, 1972.

[25] R.I. ISSA, J. Comput. Phys. 62 (1985) 40-65.

[26] R.I. ISSA, A. BRefruI, K.R. Beshay y A.D. Gosman, J. Comput. Phys. 93 (1991) 388-410. 